\title{
Apropiación y Sentido de Pertenencia en el Espacio Público: Parque Estoril (SEVILLA) ${ }^{1}$
}

\author{
Appropriation And Sense Of Belonging In Public Space: Parque Estoril \\ (SEVILLE)
}

\section{Luz Marina García Herrera}

Universidad de la Laguna - Espanha

lmgarcia@ull.edu.es

\section{Maria Carmen Díaz Rodríguez}

Universidad de la Laguna - Espanha

cdiazrod@ull.edu.es

\section{Antonio García}

Universidad Pablo de Olavide - Espanha agargar1@upo.es

\author{
Alejandro Armas Díaz \\ Universidad de la Laguna - Espanha \\ aarmas@ull.edu.es \\ Juan Samuel García Hernández \\ Universidad de la Laguna - Espanha \\ samuel.garcia.09@ull.edu.es
}

\section{Resumen}

El espacio público urbano es un elemento central de la vida cotidiana y puede por ello actuar como ámbito de identificación y relación afectiva. El presente trabajo, utilizando una metodología cualitativa, tiene por objeto explorar cómo los hombres y las mujeres a través de sus prácticas diarias dan un valor significativo y experimentan un sentimiento de apego por él. El análisis se enmarca en un contexto social, cultural y
Abstract

As a central element of daily life, the urban public space can work as a field of identification and emotional relationship. Using a qualitative methodology, this paper aims to investigate how women and men give a meaningful value and develop an affective attachment to it through their daily practices. The analysis is framed within a specific social, cultural, political and economic context, such as a low-income neighborhood 
económico específico: un barrio de bajos ingresos en la ciudad de Sevilla (España). La reclamación de un espacio público, el Parque Estoril, y la intervención de la comunidad en su diseño, significó una lucha para mejorar las condiciones de vida de sus residentes. Éste se ha convertido en un lugar vibrante de relaciones sociales y de pertenencia.

Palabras-clave: Espacio Público; Sentido de Pertenencia; Apropiación; Sevilla; España. in the city of Seville (Spain). The claim for a public space, the Parque Estoril, and its design by the community, meant a struggle to improve the living conditions of the residents. It has become a vibrant place for social life and also a space of belonging.

Keywords: Public Space; Sense of Belonging; Appropriation; Seville; Spain. 


\section{Vida Cotidiana, Espacio Público y Sentido de Pertenencia}

El proceso de globalización económica ha significado la transformación de las ciudades en centros financieros $y$ de toma de decisiones corporativas que, en desigual medida, organizan la producción. Esta deriva de su economía y gobernanza se ha producido en paralelo al aumento de la polarización y la diferenciación social, que han generado importantes luchas ciudadanas. En este sentido, en las ciudades se producen formas cotidianas y sutiles de contestación que tienen que ver con la creación o rechazo de un sentido o identidad de lugar, la búsqueda de actividades creativas que permitan superar la mediocridad cotidiana, o la construcción de comportamientos autónomos respecto a la 'norma', etc. (ANGUELOVSKI, 2013).

Autores como Lefebvre (1974) o De Certeau (1984) subrayaron el interés de las actividades diarias de los individuos como materia de análisis social. En la misma línea Vaiou y Lykogianni (2006, p. 732) argumentan que la vida cotidiana se despliega como parte de la dinámica urbana pues se lleva a cabo en aquellos "lugares donde las mujeres y los hombres viven, trabajan, se relacionan con los demás, forjan sus identidades, afrontan o desafían la rutina, las costumbres y los códigos de conducta establecidos". Al tiempo que destacan la escasa atención que se presta a las escalas espaciales 'por debajo' de la urbana (el barrio, la comunidad, la casa, el cuerpo), reclaman la importancia del barrio como esfera donde se desenvuelve una buena parte de la vida cotidiana.

Muchos estudios han demostrado que el barrio es un ámbito espacial importante para el desarrollo de la vida cotidiana de hombres y mujeres, así como para combatir la exclusión (MADANIPOUR, 2010; VAIOU y
LYKOGIANNI, 2006; ORTIZ, 2004), tanto desde la perspectiva de estrategias de reciclaje por la propia población, como de distintas fórmulas de gestión (HERNÁNDEZ AJA, 2000). En este trabajo consideramos pertinente la conceptualización de barrio, planteada por Vaiou y Lykogianni (2006), como un lugar de interacción social y desarrollo de vínculos locales que contribuyen a un sentido de seguridad y pertenencia, al tiempo que funciona como un lugar abierto, no espacialmente acotado, en tanto la actividad diaria se desarrolla y entrelaza a través de diferentes escalas espaciales, que abarcan desde el entorno local inmediato hasta el ámbito metropolitano o mundial.

De igual modo, el espacio público es un elemento central en la vida cotidiana porque influye de manera positiva en la calidad de vida (MADANIPOUR, 2010; ORTIZ, BAYLINA y PRATS, 2008; PADDISON y SHARP, 2007; SEGOVIA y NEIRA, 2005) y permite participar en la vida pública, aunque también puede ser un telón de fondo para el desarrollo de la discriminación y la marginación (DÍAZ-CORTÉS y GARCÍARAMÓN, 2010). Por tanto, los espacios públicos tienen una dimensión material pero, sobre todo, son lugares de relación social y abiertos, por tanto, a una diversidad de experiencias. Éstas comprenden tanto las prácticas individuales y colectivas como los distintos significados que adquieren para las personas (KASPAR y BUEHLER, 2009; GEHL, 2006; MORANDI, 1996).

La administración ha centrado su atención en los espacios públicos de localización central, buscando la integración social en unos casos, legitimar sus actuaciones en otros, o por razones de marketing en un gran número de ciudades. En cambio omite el cuidado de tales espacios en los barrios desfavorecidos que se caracterizan por su ausencia o su escaso mantenimiento, lo que 
reduce sus oportunidades de mejora social y económica. En estos barrios la relación entre los espacios públicos y la vida cotidiana de los residentes adquiere una relevancia singular pues aquellos son "esenciales para atender las necesidades diarias de los residentes proporcionando lugares para el encuentro, generando vínculos afectivos con el barrio y la ciudad, o sirviendo como una extensión de la vivienda" (MADANIPOUR, 2010, p. 121, 123 y 129). Asimismo, pueden tener una importante dimensión como espacios de orgullo y autoafirmación frente al resto de la ciudad (BOHIGAS, 1999).

También los estudios de género han destacado la importancia de los espacios públicos en la vida cotidiana de las mujeres en relación con su papel en la reproducción social, la necesidad de la interacción social y un mayor interés por la naturaleza (HARTH, 2007; ORTIZ, GARCÍA-RAMÓN y PRATS, 2004). Mediante las actividades diarias y el uso de los espacios públicos, sin olvidar los procesos reivindicativos y de lucha, las personas desarrollan un sentimiento de pertenencia y de apego a plazas y parques que está asociado a las experiencias, presentes o pasadas vividas en un lugar, además del conocimiento del entorno y la relación que establecen con otras personas (HERNÁNDEZ-BONILLA, 2010; KASPAR y BUEHLER, 2009; VAIOU y LYKOGIANNI, 2006; FENSTER, 2005). De igual modo, las experiencias y las actividades que hombres y mujeres desarrollan en los espacios públicos se entrecruzan con categorías como la clase, género, edad, identidad sexual y características étnicoculturales (VALENTINE, 2007; DAY, 2006; VAN HOVEN y HOERSCHELMANN, 2005; FENSTER, 2005; RUDDICK, 1996). Es decir, tales rasgos influyen en el uso y apropiación que los ciudadanos pueden hacer de los espacios en que desarrollan su vida cotidiana y también en los derechos que puedan adquirir en ellos (CUCURELLA, 2007; FENSTER, 2005). Asimismo, la distinción entre espacios públicos y privados atribuye la pertinencia de determinadas prácticas en los primeros, mientras otras distintas se confinan al ámbito doméstico. No obstante, estudios recientes muestran la redefinición de tales fronteras mediante las acciones colectivas de distintos grupos (PARLETTE y COWEN, 2011; DÍAZCORTÉS y GARCÍA-RAMÓN, 2010; BUEHLER, KASPAR y OSTERMANN, 2010; VAIOU y KALANDIDES, 2009; IVESON, 2003).

\section{El Método Cualitativo del Análisis}

En este trabajo se exploran los sentimientos de pertenencia y de apego entre los residentes del Parque Estoril, en el barrio del Cerro del Águila de la ciudad de Sevilla. El análisis busca conocer cuál es el papel que el parque desempeña en la vida cotidiana de sus usuarios y usuarias, así como los vínculos afectivos que se crean en este espacio público. El trabajo de campo se realiza entre octubre de 2012 y marzo de 2013, y la información se obtiene mediante: observaciones in situ, cuestionarios de preguntas abiertas y entrevistas semiestructuradas. Cada técnica aporta distintas y complementarias perspectivas sobre el proceso de producción y apropiación del parque. Las observaciones permitieron, tanto el examen de las particularidades formales del parque (accesibilidad, mobiliario, dotaciones y conservación) como de los espacios residenciales de su entorno y la identificación de los rasgos de los que frecuentaban el lugar y las prácticas desarrolladas de forma individual o grupal.

Los cuestionarios se cumplimentaron a través del contacto directo con las personas en el mismo parque, tras una selección aleatoria de los sujetos presentes en él, en 
distintas jornadas de mañana y de tarde y en días laborables y festivos. Las respuestas aportaron una primera aproximación a las razones que llevaban al uso del espacio, a las vivencias que en él tenían lugar, así como a otras particularidades relativas a su uso, ocupantes habituales y formas de relacionarse.

Por último, la fuente principal de información en este trabajo procede de las entrevistas que se efectuaron mediante un muestreo en bola de nieve, entre sujetos con edades y circunstancias vitales heterogéneas (jóvenes y mayores, recién llegados y antiguos residentes, usuarios cotidianos y esporádicos del parque), para diversificar el perfil de los entrevistados y sus opiniones ${ }^{2}$. Todas las entrevistas se grabaron y transcribieron literalmente, respetando el orden del discurso y las formas de expresión empleadas. Fueron validadas por los entrevistados $y$, finalmente, codificadas mediante la selección de los núcleos de sentido (palabras, frases o fragmentos) con significados relevantes (BUEHLER, KASPAR y OSTERMANN 2010). La información obtenida, junto a la que previamente se recoge de distintos estudios sobre el barrio, ha permitido reconstruir cómo era el entorno en el que se gestó la iniciativa de 'crear el Parque Estoril', cómo se llevó a cabo su conquista y diseño, y cuáles son las vivencias y los sentimientos que los entrevistados tienen del parque y el barrio en el que se inserta.

\section{El Parque Estorill: la Apropiación de un Espacio Público}

El análisis del proceso de apropiación del Parque Estoril tiene el interés de ser un caso significativo de la 'conquista' de un espacio público por parte de los residentes de su entorno que buscaban mejorar así sus condiciones cotidianas de vida
(MADANIPOUR 2010; SEGOVIA y NEIRA, 2005). Investigar la construcción social de ese parque es una manera de expresar la relevancia que tienen estos territorios comunes para los ciudadanos, tal y como han revelado otros análisis sobre los movimientos vecinales de defensa o reivindicación de estos lugares colectivos (AGUELOVSKI, 2013; GARCÍA FERRER, 2012).

En la planificación y construcción del espacio público de la ciudad de Sevilla en los últimos decenios ha primado la estrategia de gestión de arriba abajo, aunque existen casos de interés de procesos a la inversa. Respecto a las periferias urbanas ha sido más importante la atención a parques y espacios verdes de mayores dimensiones, mientras el tratamiento más individualizado de plazas y espacios públicos de proximidad se ha desarrollado fundamentalmente en las zonas centrales de la ciudad (GARCÍA, 2011).

El barrio en el que se inscribe el Parque Estoril, situado en la zona Este de Sevilla, se origina a partir de la parcelación de terrenos rústicos durante el primer tercio del siglo $\mathrm{XX}$ (ALMUEDO, 2009; ALMOGUERA Y HERNÁNDEZ, 1999). Su carácter de barrio autoconstruido explica su escasez de zonas verdes; un hecho que, a comienzos de la pasada década, percibía el $80 \%$ de sus residentes frente al $47 \%$ de los del municipio sevillano (MINISTERIO DE FOMENTO, 2011). Una nota distintiva del Cerro del Águila es que sus habitantes han desarrollado lazos de convivencia y amistad, que contribuyen al sentimiento de identidad 'ser cerreño' - , que muchos manifiestan, en particular los que residen en el entorno del parque. Un hecho, por lo demás, común en asentamientos de origen similar, como refieren otros análisis de caso en España (DÍAZ-CORTÉS y GARCÍA-RAMÓN, 2010).

La lucha por la apropiación de este 
espacio se prolongó durante más de una década (1989 - 2005); fue, por tanto, un proceso largo y difícil que explica, en parte, el aprecio que los vecinos han desarrollado hacia este lugar de encuentro y esparcimiento para muchos habitantes del barrio, que ellos diseñaron $y$ que gestionan desde su inauguración en 2007.

La construcción del parque empieza a gestarse a finales de la década de 1980 en un enclave físicamente degradado, un solar baldío, que servía de campo de futbol improvisado y que la gente percibía como amenazador, pues era poco frecuentado, oscuro y asociado al tráfico de drogas, la violencia y el vandalismo. El deseo de una parte de los residentes de disponer de un lugar donde jugaran sus hijos y fuera posible compartir y relacionarse entre ellos, se materializó en diversas estrategias para ocuparlo y conquistarlo. Las actuaciones que emprendieron para reivindicar su uso, se mantienen vivas en el recuerdo: “ [...] esto era el Barrio Chino, venían a comprar droga, peleas de perros continuamente, esto las candelas por las noches, todo eso teníamos nosotros [...]" (Cristina, residente durante 23 años, 16/12/2012).

\section{[...] al principio, nosotros íbamos y} hablábamos, oye que no se puede hacer fuego aquí, [...] cómo que no hacemos fuego, aquí hacemos fuego cuando queramos [...] hasta que ya dijimos, esto no puede ser, y salíamos con cubos de agua, [...] yo les tiraba el cubo de agua al fuego, [...] como apagues el fuego te vamos a dar una paliza, te vamos a matar, [...] Al principio íbamos un poco con miedo porque no sabíamos la reacción de esta gente [...] al final eso se quedaba en paz. (Jorge, residente durante 18 años, 15/12/2012).
Una de las fórmulas para su ocupación consistió en acudir habitualmente con los niños hasta convertir esa práctica diaria en una rutina con la que, poco a poco, transformaron un espacio peligroso en otro público, donde era posible la presencia de usuarios diversos. El acto cotidiano de sentarse con los hijos jugando alrededor, era una manera de reclamar y expulsar a la vez a los grupos vinculados a las drogas, tal y como ellos relatan: "utilizábamos mucho los niños para reivindicar el espacio, y llamábamos a los vecinos, oye vamos a bajar con los niños [...]" (Felipe, residente durante 23 años, 16/12/2012); "yo mientras ellas (sus hijas) estaban fuera yo estaba con ellas fuera porque queríamos ocupar ese espacio [...] lo importante era que esta gente, $[\ldots]$ se dieran cuenta de que [...] yo tengo derecho a este lugar público y lo voy a ocupar (Jorge, residente durante 18 años, 15/12/2012).

En este proceso de apropiación y de territorialización, las mujeres jugaron un papel muy destacado, pues: "[...] básicamente eran las mujeres, normalmente las mujeres y tal eran las primeras pero los hombres iban acompañando el tema" (Teresa, residente durante 33 años, 25/01/2013). De hecho, su defensa como espacio de vida cotidiana ha estado liderada por una mujer que, en su papel de presidenta de la asociación de vecinos del Parque Estoril, impulsa desde el comienzo hasta la actualidad la movilización vecinal.

Un activismo que consiguió cambiar, cuando se elaboraba el Plan General de Ordenación de Sevilla de 2006, la calificación del suelo, de residencial a zona verde. Ese logro consolida los sentimientos de apego, que se refuerzan cuando consiguen imponer su proyecto de parque 'integral', es decir, orientado a la mejora física del espacio $\mathrm{y}$ al enriquecimiento cultural, educativo y recreativo de sus usuarios. Hoy el parque es un recinto de $9.700 \mathrm{~m} 2$, en el que se 
combinan variados usos: parque infantil, huerto-jardín, fuente central, áreas deportivas $\mathrm{y}$, lo más importante, donde se realizan actividades comunitarias (reuniones, celebraciones, mercadillos, fiestas locales... etc.), iniciadas mucho antes de su finalización e inauguración. Es decir, esas prácticas sociales son, asimismo, parte del proceso de construcción del parque (LEFEBVRE, 1974; LOW, 2000). Mediante estos actos participativos los residentes adquieren visibilidad, se identifican y crean un lugar cargado de significados para ellos: "este olivo lo plantamos todo el mundo, todo el mundo le echamos una poquita de arena, el símbolo de la inauguración del parque [...]" (Cristina, residente durante 23 años 16/12/2012).

Entre las celebraciones de mayor relevancia destaca la Velá, una fiesta lúdicoreligiosa que cada año, desde 2000 , celebran en el parque: "nosotros cuando hicimos la primera Velá, o sea, la Velá de El Cerro siempre se ha hecho en el Cerro y nosotros quisimos hacer algo distinto, el escenario nuestro" (Jorge, 15/12/2012). Con esta y otras muchas actividades de diversión colectiva para niños y mayores se consiguen estrechar las relaciones entre los residentes del entorno y entre éstos y el lugar, al que ya consideran una parte muy importante de sus vidas cotidianas.

\section{El Sentido de Pertenencia y de Apego al Espacio Publico}

Con las prácticas descritas de apropiación y construcción del parque se ha producido una redefinición del espacio público que ha supuesto para los residentes una prolongación de sus espacios de vida privados (BUEHLER, KASPAR y OSTERMANN, 2010; VAIOU y KALANDIDES, 2009). Tal es así, que no solo organizan comidas colectivas o celebran los cumpleaños de sus hijos, sino que también, en el verano, el parque es un lugar de convivencia vecinal entre los residentes próximos que sacan bebidas de sus casas para compartirlas en grupo. Las descripciones demuestran, tanto las variadas formas de usar el espacio y la vitalidad que puede alcanzar el parque, como el mundo de vivencias compartidas: "[...] que la gente sienta que hay vida aquí, de una forma o de otra [...] oye vamos a hacer un día un concurso de tortillas, vamos a hacer una barbacoa, y se hace" (Jorge, residente durante 18 años 15/12/2012).

He visto también más familias que llega el cumpleaños, abren la mesita nos ponemos aquí a celebrar el cumpleaños de mi hija [...] y gente también que viene mucha al parque, pues esta noche vamos a celebrar el día de yo no sé qué, y abren las mesas y se ponen ahi a comer (Ana, residente durante 9 años, 27/01/2013).

Las entrevistas revelan, además, fuertes vínculos entre el espacio público y el ámbito doméstico: el parque es considerado por las mujeres y los hombres como una extensión de su vivienda: " $[\ldots]$ pero bueno digo también los jardines tienen que formar parte del palacio, que es mi puerta, [...] pues como no lo vamos a cuidar si a mí me importa más eso que mi casa" (Cristina, residente durante 23 años, 16/12/2012). Muchos dicen sentirse tan cómodos en él, como en casa, de modo que adquiere una dimensión afectiva $\mathrm{y}$ simbólica (MADANIPOUR, 2010). De hecho, cuando se refieren a él no lo perciben como un espacio distinto al privado, sino como parte de él: "El parque es como tu casa, tú no puedes dejar de estar siempre encima, tú dejas una grietita y se te cae la pared abajo, tenemos que estar siempre encima" (Daniel, residente durante 36 años, 
17/12/2012).

Mi casa, mi casa, es que el parque nosotros lo consideramos parte nuestra [...]. Yo digo esa es mi casa, ahí vivo yo, yo vivo en la plaza, [...], nosotros vivimos donde está la plaza, la plaza Estoril, ahí vivimos nosotros [...] (Norberto, residente durante 8 años, 16/12/2013)

La identificación emocional determina que las personas valoren el parque como parte de su vida: "Pues para mí es parte de mi vida ya, [...]. Yo sé que ha habido muchas cosas malas pero lo veo más bien lo positivo que lo negativo (Celina, residente durante 33 años, 26/01/2013). Se trata, por tanto de un espacio vivido, donde se disfruta, pero también de lucha y resistencia (LEFEBVRE, 1974).

Por último, tanto los residentes tradicionales como los recién llegados que viven en los alrededores, han desarrollado relaciones de vecindad y de amistad, a través de las que expresan formar parte de un entorno social familiar: "[...] sí, sí, nos cuidamos unos a otros, y yo me siento muy arropada, yo sé que de noche si pasa algo ellos están pendientes nada más que con tocar el tabique o la puerta, a mí me satisface eso mucho" (Celina, residente durante 33 años, 26/01/2013).

[...] esto es un barrio, pero parece más un pueblecito que un barrio, aquí todo el mundo te conoce, aquí casi todos somos familia [...] somos como una piña, que aunque no seamos todos amigos pero nos conocemos de un hola, un adiós, (Marta, residente durante toda su vida, 17/12/2012).

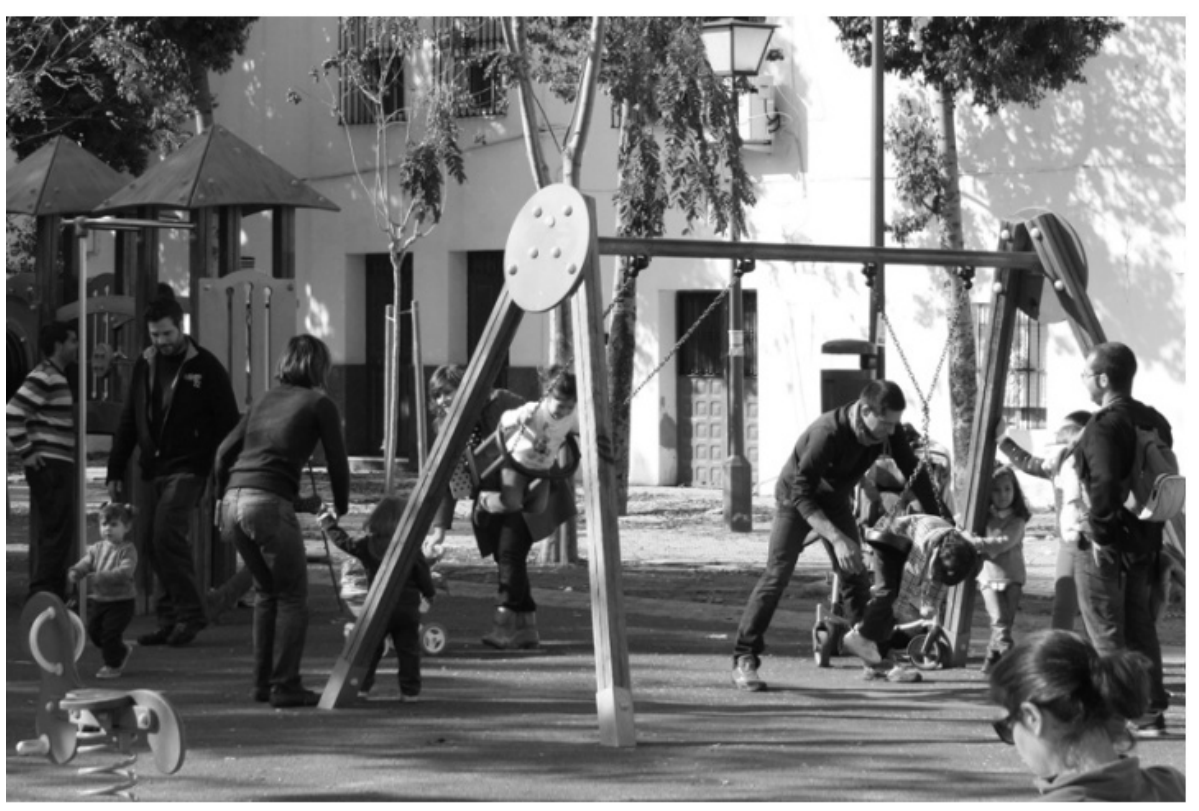

Fotografía 1.

Fuente: Los autores.

\section{Conclusiones}

El proceso de apropiación expuesto muestra cómo las prácticas cotidianas de las personas en un espacio lo van dotando de diversos significados individuales y sociales, que comprenden desde un sentido de participación reivindicativa a unos profundos lazos de copertenencia. En ese proceso, en el que las mujeres desempeñaron un papel muy relevante, destaca positivamente la construcción y defensa ciudadana de un espacio público, que se convierte en un elemento clave para la mejora de la vida diaria de los vecinos de los alrededores del parque; de modo que ese espacio de vida cotidiana es uno de los más valorados del barrio, junto a otros como la iglesia o el mercado.

\section{Luz Marina García Herrera, Maria Carmen Díaz Rodríguez, Antonio García García,} Alejandro Armas Díaz e J uan Samuel García Hernández 
La dinámica social del Parque Estoril revela asimismo la importancia del sentido del lugar. Las actividades compartidas han promovido la participación y han reforzado los vínculos sociales, convirtiendo el parque en un sitio de encuentros diarios para personas con diferentes rasgos sociales y culturales. Así los vecinos que habitan en sus alrededores han desarrollado un destacado sentimiento de pertenencia y de apego al lugar, de tal modo que para muchos el parque es un espacio que forma parte de sus vidas y hogares.

En contraposición a las difíciles condiciones de vida en un barrio con carencias importantes, a las que se añaden las precarias situaciones laborales, en muchos casos, los significados que los vecinos han ido incorporando al parque lo han convertido, parafraseando a Merrifield (2006), en un ámbito familiar, en la esfera donde se entrecruzan el espacio público y el privado, lo doméstico con el ocio, el escenario de la familia y de los amigos.

En resumen, la dimensión afectiva y las relaciones de amistad y vecindad se han forjado $\mathrm{y}$ fortalecido en el proceso de reivindicación y de territorialización de un espacio 'integral' que, como tal, atiende, al bienestar en sus aspectos materiales $y$ emocionales, lo que constituye uno de los rasgos principales de este espacio público.

1 El trabajo forma parte del Proyecto de Investigación "Ciudad y calidad de vida. El uso social de los espacios públicos abiertos en ciudades españolas" (CSO 2010-19007) financiado por el Plan Nacional de $\mathrm{I}+\mathrm{D}+\mathrm{i}$ del Ministerio de Ciencia e Innovación.

2 Se realizaron 17 entrevistas (10 a vecinos/as y 7 a otros actores clave residentes o no en el barrio) a personas distintas a las que respondieron al cuestionario.

\section{Referencias}

ALMOGUERA SALLENT, Pilar; HERNÁNDEZ RAMÍREZ, Javier. El Cerro del Águila. De periferia a ciudad. Sevilla: Ayuntamiento de Sevilla, 1999.

ALMUEDO PALMA, José. Al este del edén. Estudio demográfico del crecimiento urbano en el sector oriental de Sevilla: Nervión, Ciudad Jardín, Cerro del Águila y Amate (1922-1935). Sevilla: Ayuntamiento de Sevilla, 2009.

ANGUELOVSKI, Isabelle. Beyond a livable and green neighborhood: asserting control, sovereignty and transgression in the Casc Antic of Barcelona. International Journal of Urban and Regional Research, v. 37, n. 3, p. 1012 - 1034, 2013.

BOHIGAS, Oriol. La ciudad como espacio proyectado. En: CAPUTO, Paolo (Coord.). La arquitectura del espacio público. Formas del pasado, formas del presente. Sevilla: Triennale di Milano, Junta de Andalucía, 1999, p. 21 - 24.

BÜEHLER, Elisabeth; KASPAR, Heidi; OSTERMANN, Frank. Soziale nachhaltige Parkanlagen. Forschungsberich des Nationales Forschungsprogramms NFP 54, Zurich, 2010. Disponible en: $<$ http://www.vdf.ethz.ch/service/3309/3309 Sozial-nachhaltige-Parkanlagen_OA.pdf $>$ Consultado el 13 de Abril de $20 \overline{13}$.

CUCURELLA, Ariadna. La perspectiva de gènere en el disseny i l'ús d'espais públics urbans: el cas del Parc dels Colors de Mollet del Vallès (Barcelona). Documents d'Anàlisi geogràfica, n. 49, p. 119 - 138, 2007.

DAY, Kristen. Being feared: masculinity and race in public space. Environment and 
planning A, n. 38, p. $569-586,2006$.

DE CERTEAU, Michel. The Practice of Everyday Life. Berkeley: University of California Press, 1984.

DÍAZ-CORTÉS, Fabià; GARCÍA-RAMÓN, Maria Dolors. Mujeres, vida cotidiana y espacios públicos en la región metropolitana de Barcelona. El caso de $\mathrm{Ca}$ n'Anglada de Terrasa. Finisterra, v. 14, n. 90, p. 49 - 69, 2010.

FENSTER, Tovi. The right to the gendered city: different formations of belonging in everyday life. Journal of Gender Studies, v. 14, n. 3, p. 217 - 231, 2005.

GARCÍA FERRER, Raúl. La ciudad también se vive en los jardines. Una aproximación etnográfica a los interiores de manzana de Barcelona. Gazeta de Antropología, v. 28, n. 1, artículo 18, 2012.

GARCÍA GARCÍA, Antonio. La calle a escena. El sistema de espacio público de Sevilla y su entorno metropolitano, retos y posibilidades. Sevilla: Ayuntamiento de Sevilla, 2011.

GEHL, Jan. La humanización del espacio urbano. La vida social entre los edificios. Barcelona: Reverté, 2006.

HARTH, Annette. Open Space and Gender Gender-Sensitive Open-Space Planning. German Journal of Urban Studies [en línea], v. 1, 2007. Disponible en: $<$ http://www.difu.de/publikationen/openspace-and-gender-gendersensitive-openspace.htm>. Consultado el 13 de Abril de 2012.

HERNÁNDEZ AJA, Agustín. Barrios y equipamientos públicos, esencia del proyecto democrático de la ciudad. Documentación social, n. 119, p. 79 - 94, 2000.

HERNÁNDEZ-BONILLA, Mauricio. Making public space in low-income neighborhoods in Mexico. En: MADANIPOUR, Ali (Ed). Whose Public Space? International case Studies in Urban Design and Development. Oxford: Routledge, 2010, p. 191 - 211.

IVESON, Kurt. Justifying exclusion: The politics of public space and the dispute over access to McIvers ladies' baths, Sydney. Gender, Place \& Culture: A Journal of Feminist Geography, v. 3, n. 10, p. 215 228, 2003.

KASPAR, Heidi; BUEHLER, Elisabeth. Planning, design and use of the public space Wahlenpark (Zurich, Switzerland): functional, visual and semiotic openness. Geographica Helvetica, v. 1, n. 64, p. 21 29, 2009.

LEFEBVRE, Henri. La production de l'espace. París: Anthropos, 1974.

LOW, Setha. On the plaza: the politics of public space and culture. Austin: University of Texas Press, 2000.

MADANIPOUR, Ali. Marginal public spaces in European cities. En: MADANIPOUR, Ali. (Ed). Whose Public Space? International case Studies in Urban Design and Development. Oxford: Routledge, 2010, p. $111-130$.

MERRIFIELD, Andy. Henri Lefebvre. A Critical Introduction. Nueva York: Routledge, 2006.

MINISTERIO DE FOMENTO GOBIERNO DE ESPAÑA. Atlas de la vulnerabilidad 
urbana en España. Portal de suelo y políticas urbanas, 2011. $<\mathrm{http}$ ://siu.vivienda.es/portal/index.php?optio $\mathrm{n}=\mathrm{com}$ content\&view $=$ article \&id $=103 \&>$ Consultado el 29 de diciembre de 2012.

MORANDI, Maurizio. La città vissuta. Significati e valori dello spazio urbano. Florencia: Alinea, 1996.

ORTIZ-GUITART, Anna. Sens du lieu pour les femmes d'un quartier multiculturel de Barcelone (El Raval). Espace populations sociétés [en línea], v. 1, 2004. Disponible en: $<$ http://eps.revues.org/index529.html $>$.

Consultado el 30 de Diciembre de 2012.

ORTIZ, Anna; BAYLINA, Mireia y PRATS, Maria. Paisatges quotidians i diversitat social i de gènere: la seva relació amb la salut i el benestar. En: NOGUÉ, Joan; PUIGBERT, Laura; BRETCHA, Gemma (Eds.). Paisatge i salut. Olot: Observatori del Paisatge de Catalunya, 2008, p. 288 - 310.

ORTIZ, Anna; GARCÍA.-RAMÓN, Maria Dolors; PRATS, Maria. Women's use of public space and sense of place in The Raval (Barcelona). Geojournal, v. 61, p. 219 - 227, 2004.

PADDISON, Ronan; SHARP, Joanne. Questioning the end of public space: Reclaiming control of local banal spaces. Scottish Geographical Journal, v. 123, n. 2, p. 87 - 106, 2007.

PARLETTE, Vanessa; COWEN, Deborah. Dead Malls. Suburban activism, local spaces, global logistics. International Journal of Urban and Regional Research, v. 35, n. 4, p. $794-811,2011$.

RUDDICK, Susan M. Constructing difference in public spaces: race, class and gender as interloking system. Urban Geography, v. 17, n. 2, p. 132 - 151, 1996.

SEGOVIA, Olga; NEIRA, Hernán. Espacios públicos urbanos: una contribución a la identidad y confianza social y privada. Revista INVI, v. 20, n. 55, p. 166 - 182, 2005 .

VAIOU, Dina; KALANDIDES, Ares. Cities of 'others': public space and everyday practices. Geographica Helvetica, v. 64, n. 1, p. 11 - 20, 2009.

VAIOU, Dina; LYKOGIANNI, Rouli. Women, neighbourhoods and everyday life. Urban Studies, v. 43, n. 4, p. 731 - 743, 2006.

VALENTINE, Gill. Theorizing and Researching Intersectionality: A Challenge for Feminist Geography. The Professional Geographer, v. 59, n. 1, p. 10 - 21, 2007.

VAN HOVEN, Bettina; HÖERSCHELMMAN, Kathrin (Eds.). Spaces of Masculinities. Abingdon: Routledge, 2005.

Recebido em 18 de fevereiro de 2014. Aceito em 15 de junho de 2014. 